\title{
Managing Immune Thrombocytopenic Purpura in infants: is it time to think of Eltrombopag?
}

\author{
Giulia Ceglie ${ }^{1}$, Giulia Nocentini ${ }^{2}$, Francesca de Gennaro ${ }^{2}$, Vitangelo Clemente ${ }^{2}$, Margherita \\ Di Mauro ${ }^{2}$, and Giuseppe Palumbo ${ }^{1}$ \\ ${ }^{1}$ Bambino Gesù Pediatric Hospital Oncohematology Department of Cell Therapy Gene \\ Therapies and Hemopoietic Transplant \\ ${ }^{2}$ Ospedale Pediatrico Bambino Gesù
}

February 21, 2021

\begin{abstract}
We report 2 cases of infants with acute and persistent Immune Thrombocytopenic Purpura (ITP) treated with Eltrombopag. Since ITP is rare in infants, robust evidence about how to treat these patients is not available. Both children underwent multiple lines of treatment without success and were successfully managed with off-label use of Eltrombopag. We did not observe any of the reported adverse effects of the drug and complete remission was achieved in both cases. In one child, we were able to discontinue treatment without any ITP relapse. This is the first report of an off-label use of Eltrombopag in infants.
\end{abstract}

Title Page

Managing Immune Thrombocytopenic Purpura in infants: is it time to think of Eltrombopag? Giulia Ceglie ${ }^{1,2^{*}}$, Giulia Nocentini ${ }^{3 *}$, Francesca De Gennaro ${ }^{3}$, Vitangelo Clemente ${ }^{3}$, Margherita Di Mauro ${ }^{3}$, Giuseppe Palumbo ${ }^{1,3}$

${ }^{1}$ Department of Pediatric Hematology and Oncology, Cell and Gene Therapy, IRCCS Bambino Gesù Children's Hospital, Rome, Italy

2 PhD Program in Immunology, Molecular Medicine and Applied Biotechnology, University of Rome Tor Vergata, 00133 Rome, Italy

${ }^{3}$ University Department of Pediatrics, Bambino Gesù Children's Hospital, University of Rome Tor Vergata, Rome, Italy

*These authors contributed equally.

Correspondence : Giulia Ceglie, M.D., Department of Pediatric Hematology and Oncology, Cell and Gene Therapy, IRCCS Bambino Gesù Children's Hospital, Piazza Sant'Onofrio 4, 00165, Rome, Italy

\section{Word Count:}

Abstact: 99

Main Text: 1230

Number of Figures: 2

Running Title : Eltrombopag in 2 Infants Cases 
Keywords: Immune Thrombocytopenic Purpura (ITP), Eltrombopag, Infant Age, Thrombocytopenia, Offlabel use

Abbreviation Table :

\begin{tabular}{ll}
\hline ITP & Immune Thrombocytopenia Purpura \\
\hline QoL & Quality of Life \\
TPO-RAs & Thrombopoietin-Receptor Agonists \\
B.A.b.s. & Buchanan-Adix bleeding score \\
IVIg & Intra-Venous Immune-globuline \\
\hline
\end{tabular}

\begin{abstract}
We report 2 cases of infants with acute and persistent Immune Thrombocytopenic Purpura (ITP) treated with Eltrombopag. Since ITP is rare in infants, robust evidence about how to treat these patients is not available. Both children underwent multiple lines of treatment without success and were successfully managed with off-label use of Eltrombopag. We did not observe any of the reported adverse effects of the drug and complete remission was achieved in both cases. In one child, we were able to discontinue treatment without any ITP relapse. This is the first report of an off-label use of Eltrombopag in infants.
\end{abstract}

IntroductionImmune Thrombocytopenia Purpura (ITP) is an immune-mediated disorder characterized by isolated thrombocytopenia (platelet count $<100.000 /$ microL), with or without bleeding signs in an otherwise healthy patient [1]. It is one of the most common acquired bleeding disorders (incidence: 5-10/100.000 children/year) [2]. ITP can be classified as acute, when it is within three months from the onset; persistent, when the disease was diagnosed in the time interval between the previous 3 months and 1 year; and chronic, when it persists longer than one year. A large case series of children with ITP from the registry of the Intercontinental Childhood ITP Study Group showed that the vast majority of patients $(69.1 \%)$ had 1 to 10 years of age, only a small percentage of patients $(7.6 \%)$ were younger than 1 year [3]. Since ITP rarely presents in children younger than 1-year, robust evidence about how infants should be managed is scarce. In the pediatric setting, multiple factors besides the mere platelet count must be evaluated to decide whether to treat the patient. Thus, scoring the clinical severity through objective bleeding risk scales helps decide between a "watch and wait" approach or a pharmacological intervention. Regarding children with persistent/chronic ITP, a "watch-and-wait" approach is frequently chosen, and rescue therapies can be administered. Nevertheless, living with a low platelet count can severely impact the quality of life (QoL) of children and their families[4]; in these cases an alternative strategy should be considered, such as Thrombopoietin-Receptor Agonists (TPO-RAs) [5].

TPO-RAs stimulate platelet production and can influence platelet antibody production and $\mathrm{T}$ regulatory cell count [6-8]. Currently two TPO-RAs, Eltrombopag and Romiplostim, have been licensed for children $>$ 1-year-old with ITP lasting longer than 6 months with unsatisfactory response to standard therapies [7].

Here we report two infants presenting, respectively, with acute and persistent ITP and who were both managed with off-label use of Eltrombopag.

\title{
Case 1
}

A 3-month-old baby girl was referred to our hospital for thrombocytopenia. On admission, her platelet count was 12.000/microL and she had a Buchanan-Adix [9] bleeding score (B.A.b.s.) of 1 (isolated cutaneous petechiae). She was administered 2 doses of IVIg $(800 \mathrm{mg} / \mathrm{Kg})$, without success, hence it was decided to proceed with steroids as second line therapy (Prednisone $2 \mathrm{mg} / \mathrm{kg}$ for two weeks followed by three consecutive boluses of Methylprednisolone $30 \mathrm{mg} / \mathrm{kg}$ each). A bone marrow examination was performed before starting the steroid consistent with the diagnosis of ITP. Unfortunately, the prednisone-based therapy was unsuccessful (platelet count after 8.000/microL; B.A.b.s.=3). 
Given the unresponsiveness to glucocorticoid and to prevent steroid-related side effects it was decided to discontinue the therapy and start a close watch-and-wait approach.

At 3 months from diagnosis with a platelet count below 15.000/microL and persistent symptoms, after having obtained the informed consent from the parents, we started an off-label course of Eltrombopag (dose: 1.5 $\mathrm{mg} / \mathrm{Kg} /$ day). The first clinical response was observed after three weeks with decreasing symptoms (B.A.b.s. $0-1$ ). After about six weeks platelet count raised to $67.000 /$ microL and, 9 months from the onset, it reached a value of $164.000 /$ microL.

The patient is currently in optimal clinical condition at 11 months of follow-up and the complete blood count is normal. The current dose of Eltrombopag is $1.1 \mathrm{mg} / \mathrm{Kg} /$ die, no side effect has developed (Figure 1).

\section{Case 2}

A 5-month-old boy was admitted to our hospital following the abrupt onset of diffuse cutaneous petechiae, conjunctival hemorrhage and epistaxis (B.A.b.s. 3). Blood exams showed thrombocytopenia (platelet count $3.000 / \mathrm{microL})$. The baby was promptly started on IVIg $(800 \mathrm{mg} / \mathrm{Kg} / \mathrm{dose}$ for two consequent days) without success (platelet count $15.000 / \mathrm{microL}$ ).

Bone marrow examination was performed confirming a diagnosis of ITP and steroids were started (Prednisone $2 \mathrm{mg} / \mathrm{kg} /$ die for two weeks followed by three consecutive days of Methylprednisolone at $30 \mathrm{mg} / \mathrm{Kg} / \mathrm{dose}$ ) without success (platelet count 5.000 micro/L) and persistent symptoms. Since no clinical response was obtained, we suggested an off-label use of Eltrombopag. The parents' consent was obtained.

Clinical response was observed after one month of treatment (B.A.b.s. 0-1) and, five weeks later, platelet count started to rise (platelet count 127.000/microL) just a few days before hitting three-months from the onset.

After five weeks from platelet count normalization Eltrombopag was tapered down to $0.7 \mathrm{mg} / \mathrm{kg} / \mathrm{die}$; the following month the dose was further reduced and finally, one month later, Eltrombopag was suspended.

The patient is currently at 9 months of follow-up from ITP onset and with a normal platelet count (367.000/microL) (Figure 2).

DiscussionITP rarely presents in infants and, to the best of our knowledge, these are the first two reported cases of children younger than 1-year successfully treated with Eltrombopag. According to ASH guidelines, the treatment of choice in patients with ITP requiring medical intervention is IVIg [5][10] . Compared to steroids, IVIg elicit a faster response [11] but neither of them guarantee a long term remission[6]. In the reported cases IVIg was the first-line treatment; having obtained no response, both children were started on a course of steroids, also without success.

Steroids are well known to have significant side effects including growth impairment, weight gain, mood disturbances, osteopenia, and other metabolic effects [6]. Considering the very young age of our patients, these side effects were even more concerning [12]. In consideration of the extremely low platelet count, the persistence of symptoms and the significant impact on the QoL of these patients we started both infants on Eltrombopag.

The efficacy of Eltrombopag has been studied in two large pediatric randomized clinical trials, PETIT and PETIT2, and in a longitudinal observational study, ICON1[12]. None of these studies included children younger than 1 year of age.

The adverse events related to Eltrombopag mainly consist in reversible hepatotoxicity and iron deficiency anemia (due to the iron-chelating effect of Eltrombopag)[12].

It has also been speculated that, following the possible thrombocytosis, thromboembolic events may occur but there is currently no evidence suggesting that these are related to TPO-ARs. Similarly, increase in bone marrow reticuline and bone marrow fibrosis are theoretical - though unproven - risks of Eltrombopag treatment. Conversely, rebound thrombocytopenia is a known risk of abrupt TPO-RAs discontinuation [6, 
13]. However, because of the recent wide use of this molecule, possible long-term effects of TPO-RAs could be still unknown, and this is particularly concerning when treating very young children.

Both children had already failed two treatment lines, showed persistently low platelet count, along with bleeding symptoms. According to the approved guidelines for management, the patients would have remained off-therapy for months, during this period their motor skills development (such as crawling and walking) would have increased the risk of trauma and bleeding. These factors made it also particularly difficult to discharge them home, with a severe impact on their families QoL. The low risk of significant side effects of Eltrombopag made the decision easier to take; we carefully monitored the liver function, hemoglobin and the iron levels for both infants and no abnormalities were detected.

Currently, Eltrombopag is only available in tablets hence administering a fractioned dose can be challenging in infants; in this context, it is advisable to consider the weekly dosage over the daily one. Also, a more practical formulation in oral solution might be useful. Eltrombopag also needs not to be administered with milk and dairy products[6], making it challenging to find the right time of administration in infants. In conclusion, we report two cases of children younger than 1 year with acute and persistent ITP successfully and safely treated with Eltrombopag.

We believe further evidence is needed to define optimal dosage and drug administration in infants along with its long-term safety.

\section{Conflict of Interest Statement}

The authors received no financial support for the research, authorship, and/or publication of this article. The authors have no conflict of interest to disclose.

\section{Acknowledgments}

We thank Professor Franco Locatelli for critically reading the manuscript. We thank the association ABE (Associazione Bambini Ematoncologici) for the support.

\section{Author contributions}

GC and GN conceived the idea, researched the literature and wrote the paper, FDG, VC and MDM collected the patients' data and provided critical feedback, GP conceived the idea, provided critical feedback and supervision.

\section{References}

1. Rodeghiero F, Stasi R, Gernsheimer T, Michel M, Provan D, Arnold DM, Bussel JB, Cines DB, Chong BH, Cooper N, Godeau B, Lechner K, Mazzucconi MG, McMillan R, Sanz MA, Imbach P, Blanchette V, Kühne T, Ruggeri M, George JN. Standardization of terminology, definitions and outcome criteria in immune thrombocytopenic purpura of adults and children: report from an international working group. Blood , 2009 113: $2386-2393$.

2. Terrell DR, Beebe LA, Vesely SK, Neas BR, Segal JB, George JN. The incidence of immune thrombocytopenic purpura in children and adults: A critical review of published reports. Am J Hematol , 2010 85: $174-180$.

3. Kühne T, Buchanan GR, Zimmerman S, Michaels LA, Kohan R, Berchtold W, Imbach P, Intercontinental Childhood ITP Study Group, Intercontinental Childhood ITP Study Group. A prospective comparative study of 2540 infants and children with newly diagnosed idiopathic thrombocytopenic purpura (ITP) from the Intercontinental Childhood ITP Study Group. J Pediatr , 2003 143: 605-608.

4. Mathias SD, Li X, Eisen M, Carpenter N, Crosby RD, Blanchette VS. A Phase 3, Randomized, DoubleBlind, Placebo-Controlled Study to Determine the Effect of Romiplostim on Health-Related Quality of Life in Children with Primary Immune Thrombocytopenia and Associated Burden in Their Parents. Pediatr Blood Cancer, 2016 63: 1232-1237. 
5. Provan D, Arnold DM, Bussel JB, Chong BH, Cooper N, Gernsheimer T, Ghanima W, Godeau B, González-López TJ, Grainger J, Hou M, Kruse C, McDonald V, Michel M, Newland AC, Pavord S, Rodeghiero F, Scully M, Tomiyama Y, Wong RS, Zaja F, Kuter DJ. Updated international consensus report on the investigation and management of primary immune thrombocytopenia. Blood Adv , 2019 3: 3780-3817.

6. Grainger JD, Thind S. A practical guide to the use of eltrombopag in children with chronic immune thrombocytopenia. Pediatr Hematol Oncol , 2017 34: 73-89.

7. Neunert C, Terrell DR, Arnold DM, Buchanan G, Cines DB, Cooper N, Cuker A, Despotovic JM, George JN, Grace RF, Kühne T, Kuter DJ, Lim W, McCrae KR, Pruitt B, Shimanek H, Vesely SK. American Society of Hematology 2019 guidelines for immune thrombocytopenia. Blood Adv , 2019 3: 3829-3866.

8. Bao W, Bussel JB, Heck S, He W, Karpoff M, Boulad N, Yazdanbakhsh K. Improved regulatory T-cell activity in patients with chronic immune thrombocytopenia treated with thrombopoietic agents. Blood, 2010 116: 4639-4645.

9. Buchanan GR, Adix L. Grading of hemorrhage in children with idiopathic thrombocytopenic purpura. J Pediatr , 2002 141: 683-688.

10. Arnold SD, Brazauskas R, He N, Li Y, Aplenc R, Jin Z, Hall M, Atsuta Y, Dalal J, Hahn T, Khera N, Bonfim C, Majhail NS, Diaz MA, Freytes CO, Wood WA, Savani BN, Kamble RT, Parsons S, Ahmed I, Sullivan K, Beattie S, Dandoy C, Munker R, Marino S, Bitan M, Abdel-Azim H, Aljurf M, Olsson RF, Joshi S, Buchbinder D, Eckrich MJ, Hashmi S, Lazarus H, Marks DI, Steinberg A, Saad A, Gergis U, Krishnamurti L, Abraham A, Rangarajan HG, Walters M, Lipscomb J, Saber W, Satwani P. Clinical risks and healthcare utilization of hematopoietic cell transplantation for sickle cell disease in the USA using merged databases. Haematologica , 2017 102: 1823-1832.

11. Beck CE, Nathan PC, Parkin PC, Blanchette VS, Macarthur C. Corticosteroids versus intravenous immune globulin for the treatment of acute immune thrombocytopenic purpura in children: a systematic review and meta-analysis of randomized controlled trials. J Pediatr , 2005 147: 521-527.

12. Second-line treatments in children with immune thrombocytopenia: Effect on platelet count and patient-centered outcomes - PubMed [Internet][cited 2021 Jan 13] Available from: https://pubmed.ncbi.nlm.nih.gov/30945320/

13. Marano M, Serafinelli J, Cairoli S, Martinelli D, Pisani M, Palumbo G, Cefalo MG, Cecchetti C, Di Nardo M, Falvella FS, Goffredo BM. Eltrombopag-Induced Acute Liver Failure in a Pediatric Patient: A Pharmacokinetic and Pharmacogenetic Analysis. Ther Drug Monit, 2018 40: 386-388.

\section{Legends}

Figure 1: Case 1 Timeline

Figure 1: Case 2 Timeline 

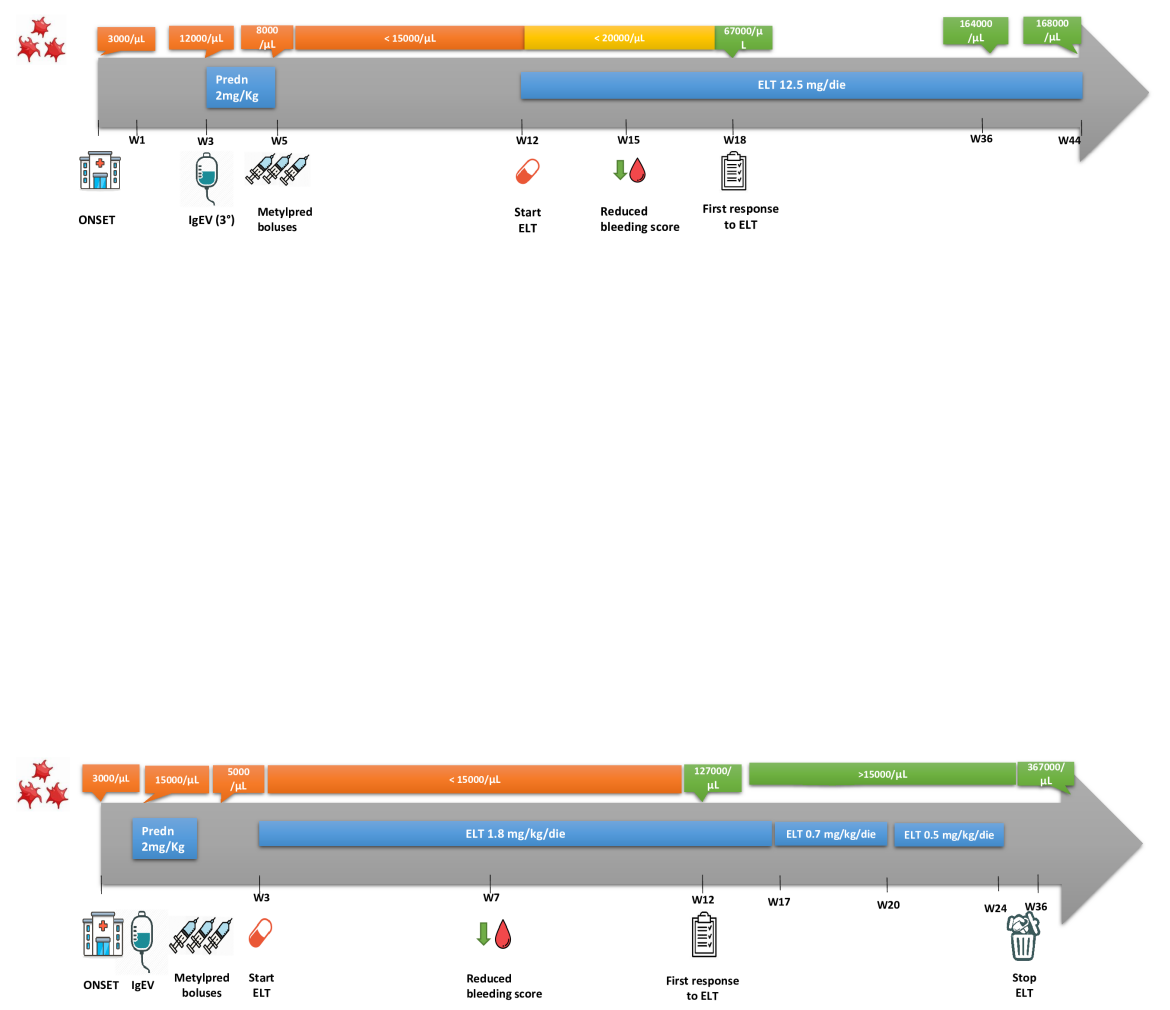